\title{
What is the Best Drainage Method for a Perinephric Abscess?
}

\author{
Ahmed R. EL-Nahas, Raed Faisal, Tarek Mohsen, Mohammed S. AL-Marhoon, Hassan \\ Abol-Enein \\ Department of Urology (AREN, RF, MSAM, HAE) and Radiology (TM), Urology and Nephrology \\ Center, Mansoura University, Mansoura, Egypt
}

\begin{abstract}
Purpose: To compare the results of percutaneous and open drainage for perinephric abscess.

Materials and Methods: The files of 86 patients who underwent drainage for perinephric abscesses from April 2001 through March 2008 were evaluated. The method of drainage for each patient was performed according to the clinical decision of the treating physician. Percutaneous tube drain (PCD) was used for drainage of the abscess in 43 patients (group 1), while the other 43 patients were managed with open drainage (group 2). Cure was defined as complete obliteration of the abscess cavity. The cure rates, complications, and hospital stay were compared between both groups.

Results: The study included 50 males and 36 females with mean age $44.2 \pm 17.3$. The most common predisposing factors were diabetes mellitus and/or stones. Open drainage of perinephric abscesses resulted in a statistically significant higher cure rate $(98 \%$ versus $69 \%, \mathrm{p}<0.001)$ and shorter hospital stay than PCD $(3.6$ versus 6 days, $\mathrm{p}<0.001)$. Failure of complete drainage of multilocular abscess was observed in 8 of 13 cases $(61.5 \%)$ in group 1 and one of 38 cases (2.6\%) in group 2 $(\mathrm{P}<0.001)$. Complications were observed in $7 \%$ of group 1 and $11.5 \%$ in group $2(\mathrm{P}=0.45)$. After mean follow-up of 19 months, 9 of 46 patients (19.6\%) had recurrence; 7 of them were in group 1.

Conclusion: Percutaneous drainage of perinephric abscess is an effective minimally invasive treatment. However, PCD is not the optimal method for drainage of multilocular abscess because open surgical drainage provided higher cure rates and shorter hospitalization than PCD.
\end{abstract}

Key words: kidney; infection; abscess; perinephric; percutaneous; surgery

Int Braz, J Urol. 2010; 36: 29-37

\section{INTRODUCTION}

Perinephric abscess (PNA) is defined as an abscess outside the renal capsule but within Gerota's fascia. They are rare in comparison to other infections involving the genitourinary tract but they can cause significant morbidity and mortality (1). Broad-spectrum antibiotic therapy is the first step in treatment and should be associated with drainage in the majority of cases. The first report of open surgical drainage of PNA was described by the French physician Germain
Colotin in 1474 (2). Surgical drainage has been the accepted practice since then. After the advent of various imaging modalities such as ultrasonography and computed tomography, percutaneous drainage (PCD) with radiological guidance has become widely used for most abdominal abscesses including selected cases of PNA (3). There have been many reported studies that have evaluated PCD as a minimally invasive treatment for PNA (3-6). Nevertheless, to our knowledge no previous series has compared PCD versus open drainage in the management of PNA. The present 
study was conducted to compare the efficacy and safety of open drainage with PCD in the management of PNA.

\section{MATERIALS AND METHODS}

The computerized files, radiographic images, operative and postoperative data of 86 patients who were treated for perinephric abscess at our center from April 2001 through March 2008 were retrospectively assessed. We included only patients with perinephric abscess who required drainage because of either presence of predisposing factors or large abscess size. Preoperative laboratory workup included serum creatinine tests, complete blood count, urinalysis and culture. Suspected patients were clinically evaluated based on detailed medical history and physical examination results. Abdominal ultrasonography was the initial radiological investigation. When the findings were suggestive of perinephric abscess, unenhanced computed tomography (CT) scan of the abdomen was performed to confirm the diagnosis and determine abscess configuration (unilocular or multilocular), volume and associated conditions (such as renal or ureteral stones).

All patients received a broad-spectrum of intravenous antibiotics (third generation cephalosporin), then PCD or open drainage was considered. The method of drainage for each patient was performed according to the clinical decision of the treating physician. PCD was chosen for severely ill patients who could not withstand general anesthesia. It was performed by a radiologist under local anesthesia using ultrasound guidance. A pigtail catheter of 12 or $14 \mathrm{~F}$ was percutaneously inserted into the abscess cavity. Open drainage was performed under general anesthesia through a small flank incision. When the abscess cavity was entered, a gentle opening of all locules was performed. This was followed by irrigation of the abscess cavity with saline and gentamycin. Two wide caliber tube drains (24F) were left indwelling.

Drainage catheters remained in place until the output was minimal and radiographic resolution was confirmed with ultrasonography. Cure was defined as complete obliteration of the abscess cavity. The patients were discharged from the hospital when they were clinically improved and free of any tubes. Patients were evaluated during follow-up visits for abscess recurrence by ultrasonography.

Percutaneous tube drain was inserted for drainage of the abscess in 43 patients (Group 1), while the other 43 patients were managed with open surgical drainage (Group 2). In 20 patients with hydronephrosis due to obstructing stones, urinary drainage with percutaneous nephrostomy tube (6) or double J stent (14) was also performed.

The cure rate, complications, hospital stay and recurrence were compared in both groups using chi-square and Student's-t-test or Mann-Whitney test.

\section{RESULTS}

The study included 50 males and 36 females with a mean age of $44.2 \pm 17.3$. Demographic data, predisposing factors and presentations are summarized in Table-1. The most common predisposing factors were diabetes mellitus and/or kidney stones. In 11 patients, the condition was associated with distant skin or subcutaneous abscess. It was observed that the success rate was $77 \%$ in diabetic patients compared with $88 \%$ in non-diabetic patients, but the difference was not statistically significant $(\mathrm{p}=0.184)$.

Preoperative laboratory and radiological data are summarized in Table- 2 .

The differences between cure rates, complications and hospital stay of group 1 and 2 are presented in Table-3. There was no significant difference between the complication rates of both groups. In group 1 , recurrent post-drainage fever was encountered in 2 patients and was controlled by intravenous antibiotics and antipyretics. In group 2, two cases of wound infection were managed by frequent dressing changes and a case of wound dehiscence required secondary sutures. Intra-operative bleeding from inadvertent inferior vena cava injury was adequately repaired with $4 / 0$ sutures and blood transfusion. Septic shock developed in one patient in each group and admission to the intensive care unit was necessary. One of them was resuscitated with cardiac inotropic drugs, intravenous fluids and antibiotics, while the other died from septic shock. 
Table 1 - Demographic data of 86 patients with perinephric abscess.

\begin{tabular}{|c|c|c|c|c|}
\hline Characteristics & $\mathbf{N}$ & $\begin{array}{c}\text { Group I (PCD) } \\
\text { N (\%) }\end{array}$ & $\begin{array}{c}\text { Group II (Open) } \\
\text { N }(\%)\end{array}$ & p Value \\
\hline Gender & & & & 0.190 \\
\hline Male & 50 & $28(56)$ & $22(44)$ & \\
\hline Female & 36 & $15(41.7)$ & $21(58.3)$ & \\
\hline Side & & & & 0.196 \\
\hline Right & 42 & $18(43)$ & $24(57)$ & \\
\hline Left & 44 & $25(56.8)$ & $19(43.2)$ & \\
\hline $\begin{array}{l}\text { Predisposing factors } \\
\text { (75 patients) }\end{array}$ & & & & 0.413 \\
\hline $\mathrm{DM}$ & 29 & $12(41.4)$ & $17(58.6)$ & \\
\hline Urolithiasis & 21 & $10(47.6)$ & $11(52.4)$ & \\
\hline Immune-compromised & 13 & $8(61.5)$ & $5(38.5)$ & \\
\hline DM + Urolithiasis & 6 & $2(33.3)$ & $4(66.7)$ & \\
\hline Open renal surgery & 6 & $5(83.3)$ & $1(16.7)$ & \\
\hline Presentation & & & & 0.610 \\
\hline Fever + loin pain & 55 & $26(47.3)$ & $29(52.7)$ & \\
\hline Loin pain & 17 & $8(47)$ & $9(53)$ & \\
\hline Fever & 10 & $7(70)$ & $3(30)$ & \\
\hline Recurrent UTI & 4 & $2(50)$ & $2(50)$ & \\
\hline
\end{tabular}

$P C D=$ percutaneous drainage; $N=$ number of cases; $D M=$ diabetes mellitus; $U T I=$ urinary tract infection.

Open drainage of perinephric abscesses resulted in statistically significant higher cure rate and shorter hospital stay than $\operatorname{PCD}(\mathrm{p}<0.001)$. Inadequate drainage was found in 17 cases of group 1; 7 of them were managed by readjustment of the PCD (replacement of the PCD with wider tube in the same locule or insertion of another PCD in other locules of the abscess) while the remaining 10 cases needed further open drainage. Inadequate drainage was observed in only one patient of group 2. This was due to missing one locule of a multilocular abscess and it was managed with PCD. The abscess configuration had a significant effect on the outcome. Failure of complete drainage of multilocular abscess was found in 8 of 13 cases $(61.5 \%)$ in group 1 and one of 38 cases $(2.6 \%)$ in group $2(\mathrm{P}<0.001)$. On the other hand, incomplete drainage of a unilocular abscess was observed in one of 29 cases ( $82 \%$ ) in group 1 (because of large abscess volume with thick pus) and none of 5 cases $(100 \%)$ in group $2(\mathrm{p}=0.315)$.

Eight patients required delayed nephrectomy for non-functioning ipsilateral kidney. After mean follow-up of 16.7 months (range 3-65), 9 out of 46 patients $(19.6 \%)$ suffered recurrence of PNA in the ipsilateral side, 7 of them were in group 1 .

\section{COMMENTS}

While urinary tract infections are common, the severe complications of renal and perinephric abscess formation are uncommon and usually occur in patients with predisposing factors such as diabetes mellitus, urinary calculi, urinary obstruction and immune compromised patients. Moreover, multiple predisposing factors may be present in the same patient (1). The same findings were observed in the present study. We identified diabetes, urolithiasis, and immune-compromised conditions as predisposing factors for PNA and in some patients multiple factors were present (Table$1)$.

Prior to the development and availability of antibiotics, most perinephric abscesses were due to hematogenous spread of gram positive bacterial 
Table 2 - Preoperative radiological and laboratory data of 86 patients with perinephric abscess.

\begin{tabular}{|c|c|c|c|c|}
\hline Characteristics & $\mathbf{N}$ & $\begin{array}{c}\text { Group I (PCD) } \\
\text { N }(\%)\end{array}$ & $\begin{array}{c}\text { Group II (Open) } \\
\mathbf{N}(\%)\end{array}$ & p Value \\
\hline Kidney status & & & & 0.186 \\
\hline Normal & 38 & $17(44.7)$ & $21(55.3)$ & \\
\hline Hydronephrosis & 21 & $14(66.7)$ & $7(33.3)$ & \\
\hline Pyelonephritic & 19 & $10(52.6)$ & $9(47.4)$ & \\
\hline Non-functioning & 8 & $2(25)$ & $6(75)$ & \\
\hline Associated renal stone & & & & 0.610 \\
\hline Yes & 20 & $9(45)$ & $11(55)$ & \\
\hline No & 66 & $34(51.5)$ & $32(48.5)$ & \\
\hline Urine culture & & & & 0.336 \\
\hline Negative & 24 & $10(41.7)$ & $14(58.3)$ & \\
\hline Positive & 62 & $33(53.2)$ & $29(46.8)$ & \\
\hline Escherichia coli & 26 & 11 & 15 & \\
\hline Klebsiella pneumonia & 14 & 12 & 2 & \\
\hline Staphylococcus aureus & 11 & 4 & 7 & \\
\hline Enterobacter & 4 & 3 & 1 & \\
\hline Pseudomonas aeruginosa & 3 & 2 & 1 & \\
\hline Candida albicans & 2 & 0 & 2 & \\
\hline Proteus mirabilis & 2 & 1 & 1 & \\
\hline Abscess configuration & & & & $<0.001$ \\
\hline Multilocular & 51 & $13(25.5)$ & $38(74.5)$ & \\
\hline Unilocular & 35 & $30(85.7)$ & $5(14.3)$ & \\
\hline Abscess volume (cc) & & & & 0.004 \\
\hline Mean & 332 & 260 & 403 & \\
\hline Range & $(40-1110)$ & $(40-810)$ & $(120-1110)$ & \\
\hline Hemoglobin (gm/dL) & & & & 0.283 \\
\hline Mean & 10.4 & 10.6 & 10.1 & \\
\hline Range & $(5.2-16.2)$ & $(7-16.2)$ & $(5.2-15.7)$ & \\
\hline Serum creatinine $(\mathrm{mg} / \mathrm{dL})$ & & & & 0.833 \\
\hline Mean & 1.97 & 2 & 1.9 & \\
\hline Range & $(0.3-9.9)$ & $(0.3-9.9)$ & $(0.6-9.5)$ & \\
\hline
\end{tabular}

$P C D=$ percutaneous drainage; $N=$ number of cases .

infection (such as Staphylococcus aureus) resulting in formation of renal cortical abscess $(7,8)$. Currently, the majority of perinephric abscesses are due to gram negative bacteria which ascend in a retrograde fashion from the lower urinary tract causing corticomedullary abscess (9). A PNA is formed when a cortical or corticomedullary abscess eventually rupture into the perinephric space. Opportunistic organisms such as Candida albicans are also isolated from immune suppressed and diabetic patients (10). In the present study, the most common isolated organisms were gram negative bacilli (such as Escherichia coli and Klebsiella pneumonia), while Staphylococcus aureus was isolated from $13 \%$ of patients.

Traditionally, PNA has been associated with significant morbidity and high rates of mortality reaching up to $56 \%$ (11). This was attributed to delay in diagnosis because the symptoms of PNA are somewhat non-specific and confusing. Due to the 
Table 3 - Comparison between group 1 (PCD) and group 2 (open) regarding outcome parameters.

\begin{tabular}{lccc}
\hline Variable & Group I (PCD) & Group II (Open) & p Value \\
& N $(\%)$ & N $(\%)$ & \\
\hline Cure rate & $29 / 42 *(69)$ & $42 / 43(98)$ & $<0.001$ \\
Complication rate & $3 / 43(7)$ & $5 / 43(11.5)$ & 0.45 \\
$\quad$ Wound complications & - & 3 & \\
$\quad$ Post-drainage fever & 2 & - & \\
$\quad$ Post-drainage septic shock & - & 1 & \\
Bleeding & $6(1-19)$ & $3.6(1-7)$ & $<0.001$ \\
Hospital stay: mean (range) & & & \\
\hline
\end{tabular}

* One patient died and was excluded; $P C D=$ percutaneous drainage; $N=$ number of cases.

introduction of cross-sectional imaging modalities such as CT, in addition to improvement of ultrasound examinations, early diagnosis and minimally invasive treatment represent major advances in management of PNA during the last 20 years.

The wide spread utilization of ultrasound for examination of patients with loin pain, fever or other non-specific complaints has resulted in early diagnosis of PNA. When there is any suspicion regarding the nature of the lesion, CT is a valuable tool for confirmation of the diagnosis (11). Therefore, the mortality rates in recent series have decreased to $12 \%$ $14 \%(1,12)$. The authors attributed mortality among their patients to medical treatment of abscesses that otherwise needed drainage. In the present study, we used ultrasound for evaluation of all patients with suspicious symptoms or predisposing factors of PNA and $\mathrm{CT}$ was used in to confirm the diagnosis. Then, we drained PNA that were large or present in patients with predisposing factors. This may be the reason for very low mortality rate (1\%) among our patients.

The classic open surgical drainage for perinephric abscess has been challenged by the introduction of image guided percutaneous tube drainage methods and antibiotics alone (12). The general consensus is that large abscesses and patients with predisposing factors require drainage in addition to antibiotics $(12,13)$. Therefore, antibiotics without drainage are suitable for selected cases and the decision to treat with antibiotics alone requires consideration of other associated medical conditions (1). The reason for not using antibiotics alone in our patients was either presence of predisposing factors or large abscess size.

Percutaneous drainage with radiological guidance has become the treatment of choice for most abdominal abscesses as it usually provides satisfactory clinical results with minimal complications, thus obviates the need for open surgery (14). Percutaneous drainage of PNA has been reported in many series (3-6). The ease of image guided fixation of PCD without the need for general anesthesia has made it the preferred choice for severely ill patients. Moreover, the cure rate of $60-67 \%$ was the main reason that made PCD the most commonly used minimally invasive intervention for treatment of PNA. The main disadvantage was the need for adjustment or insertion of multiple draining tubes in many patients $(1,3-6,13)$. In the present study, $\mathrm{PCD}$ was able to adequately drain the abscess in $60 \%$ of patients, and subsequently the cure rate was increased to $69 \%$, after adjustment of the tube in 7 patients. Another disadvantage of PCD was the longer hospital stay because of slow drainage or the need for multiple interventions.

In our study, the main cause of PCD failure was multilocular abscess cavity. The PCD can only drain the locule at its site of insertion. Therefore, it may be beneficial to fix multiple tubes from the start in multilocular abscess. On the other hand, a quick extraperitoneal open drainage provided very high cure rate $(98 \%)$ even in patients with multilocular abscess because of manually disrupting the septa between the abscess locules. Moreover, evacuation of thick pus during open drainage and wide draining tubes resulted 
in significantly shorter hospital stay. However, these achievements were gained at the expense of using a general anesthesia and a slightly higher complication rate than PCD. It is also important to emphasis that severely ill patients cannot tolerate nephrectomy of a non-functioning kidney at the time of PNA drainage.

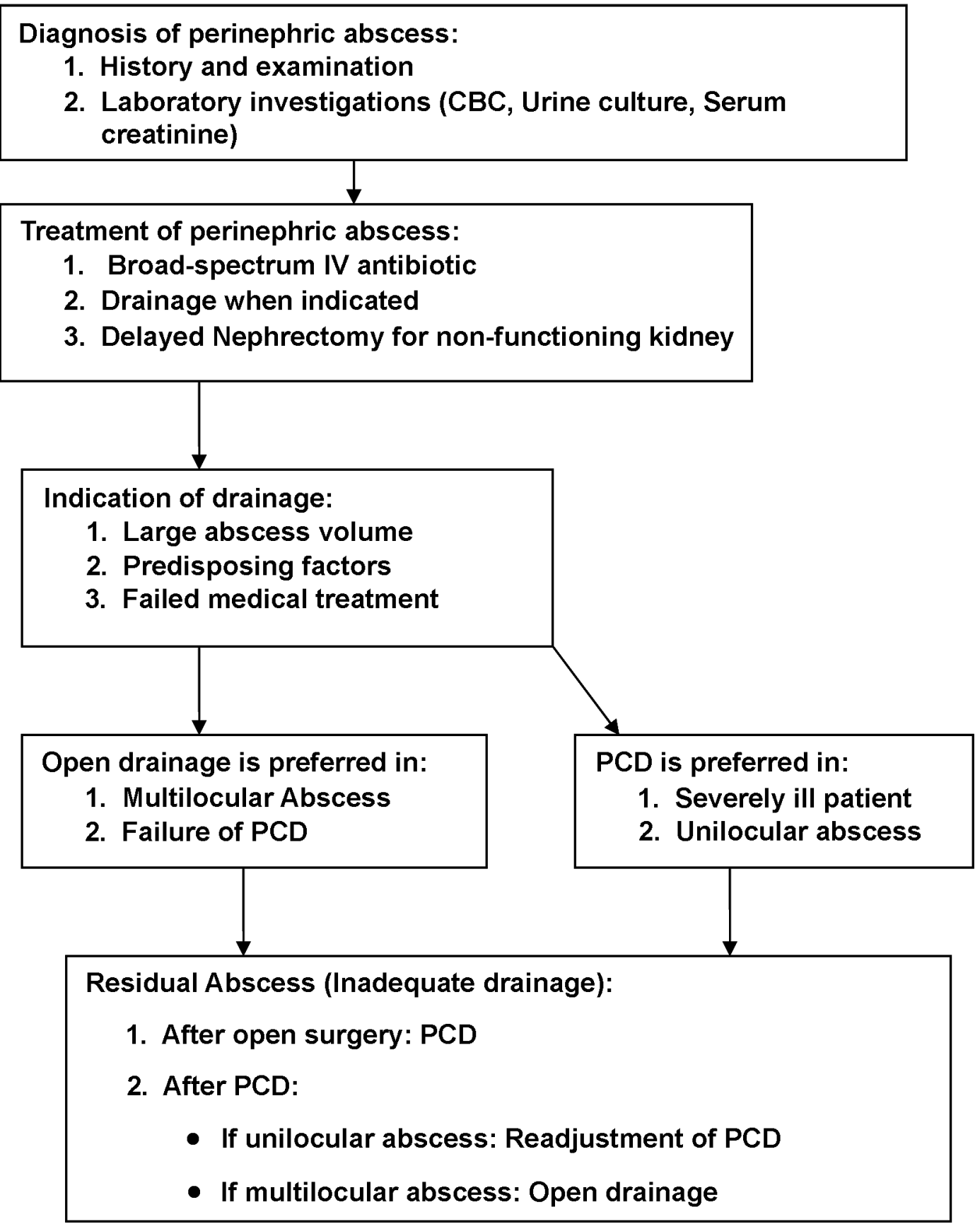

Figure 1 -Algorithm for management of perinephric abscess. $P C D=$ percutaneous tube drain; $C B C=$ complete blood count . 
Therefore, delayed nephrectomy was performed in 8 patients following complete abscess drainage and improvement of the patients' general condition.

Although the selection bias for each treatment option cannot be completely eliminated, it was statistically proven in our study that PCD was not the optimal drainage method for multilocular PNA. This was based on the lower cure rate of PCD in the treatment of multilocular PNA and the higher recurrence rate that may have resulted from enlargement of a very small residual. However, a prospective randomized trial is warranted to confirm these findings. A proposed algorithm for treatment of perinephric abscess is illustrated in Figure-1.

\section{CONCLUSIONS}

Percutaneous drainage of a perinephric abscess is an effective minimally invasive treatment modality. Therefore, when a PNA has to be drained, we recommend PCD as the primary drainage method because, as shown in our series, it can save $69 \%$ of patients an open surgery. However, and after acknowledging the limitations of the retrospective nature of the study. PCD is not the optimal method for drainage of multilocular abscess because open surgical drainage provided higher cure rates, shorter hospitalization and lower recurrence rate than PCD.

\section{ACKNOWLEDGMENT}

Dr. Mohamed Motwally assisted in data collection.

\section{CONFLICT OF INTEREST}

None declared.

\section{REFERENCES}

1. Meng MV, Mario LA, McAninch JW: Current treatment and outcomes of perinephric abscesses. J Urol. 2002; 168: 1337-40.
2. Morgan WR, Nyberg LM Jr: Perinephric and intrarenal abscesses. Urology. 1985; 26: 529-36.

3. Siegel JF, Smith A, Moldwin R: Minimally invasive treatment of renal abscess. J Urol. 1996; 155: 52-5.

4. Gerzof SG, Gale ME: Computed tomography and ultrasonography for diagnosis and treatment of renal and retroperitoneal abscesses. Urol Clin North Am. 1982; 9: 185-93.

5. Deyoe LA, Cronan JJ, Lambiase RE, Dorfman GS: Percutaneous drainage of renal and perirenal abscesses: results in 30 patients. AJR Am J Roentgenol. 1990; 155: 81-3.

6. Lang EK: Renal, perirenal, and pararenal abscesses: percutaneous drainage. Radiology. 1990; 174: 109-13.

7. Truesdale BH, Rous SN, Nelson RP: Perinephric abscess: a review of 26 cases. J Urol. 1977; 118: 910-1.

8. Jemni L, Mdimagh L, Jemni-Gharbi H, Jemni M, Kraiem C, Allegue M: Kidney carbuncle: diagnostic, bacteriological and therapeutic considerations. Apropos of 11 cases. J Urol (Paris). 1992; 98: 228-31.

9. Noble MJ: Perinephric abscess. AUA update. Series 2002; Vol. XXI, Lesson 10; 74-79.

10. Patterson JE, Andriole VT: Bacterial urinary tract infections in diabetes. Infect Dis Clin North Am. 1997; 11: 735-50.

11. Salvatierra O Jr, Bucklew WB, Morrow JW: Perinephric abscess: a report of 71 cases. J Urol. 1967; 98: 296-302.

12. Rai RS, Karan SC, Kayastha A: Renal and perinephric abscesses revisited. MJAFI 2007; 63: 223-225.

13. Coelho RF, Schneider-Monteiro ED, Mesquita JL, Mazzucchi E, Marmo Lucon A, Srougi M: Renal and perinephric abscesses: analysis of 65 consecutive cases. World J Surg. 2007; 31: 431-6.

14. vanSonnenberg E, Mueller PR, Ferrucci JT Jr: Percutaneous drainage of 250 abdominal abscesses and fluid collections. Part I: Results, failures, and complications. Radiology. 1984; 151: 337-41.

Accepted after revision:

September 18, 2009

\section{Correspondence address:}

Dr. Ahmed R. El-Nahas

Urology \& Nephrology Center

Mansoura University

Mansoura, Egypt

Fax: + 20502263717

E-mail: ar_el_nahas@yahoo.com 


\section{EDITORIAL COMMENT}

Perinephric abscess is a serious entity neglected in the urological literature. Morbidity and mortality have fallen in the last decades thanks to the progress in diagnosis with ultrasonography and overall computed tomography (CT) scan and due to improvement in medical and surgical therapies. Nevertheless, the best way of surgical management of such collections has been a subject of debate. In this article, the authors compared retrospectively the outcome of 43 perinephric abscess drained percutaneously with 43 cases drained by open surgery. Renal stones and diabetes mellitus were the most important predisposing factors. Abscesses drained by open surgery were significantly greater and multiloculated when compared to percutaneously drained abscesses. The cure rate was significantly higher in the group treated by the open access. There were no difference in the complication rate but hospital stay was longer among patients treated percutaneously.

This article concerns three important issues: A) Perinephric abscesses occur with some frequency and must be always suspected in patients with prolonged fever, overall in diabetics and in patients with stones, B) CT is an important tool and should be considered the gold-standard, not only in early diagnosis but also in the planning of the therapy to be instituted, and C) Minimally invasive therapy is not always the best option. In fact, as the authors clearly showed, a quick open drainage is more efficient especially in multiloculated collections. In this setting percutaneous drainage should be reserved for patients in a severe clinical condition, when it can be performed under local anesthesia or for uniloculated abscesses with greater chance of success.

The results corroborate those obtained by Coelho et al. (1) that also found diabetes mellitus and stones as the main predisposing factors for perinephric abscesses and by Meng et al. (2) who showed that CT could influence prognosis as it allows for earlier diagnosis and that a $36 \%$ failure rate with percutaneous drainage can be observed in such cases. Another important point is that small perinephric collections (usually less than $2 \mathrm{~cm}$ ) can be managed successfully with adequate antibiotics and correction of eventual predisposing factors like urinary obstruction (2).

\section{REFERENCES}

1. Coelho RF, Schneider-Monteiro ED, Mesquita JL, Mazzucchi E, Marmo Lucon A, Srougi M: Renal and perinephric abscesses: analysis of 65 consecutive cases. World J Surg. 2007; 31: 431-6.

2. Meng MV, Mario LA, McAninch JW: Current treatment and outcomes of perinephric abscesses. J Urol. 2002; 168: 1337-40.

Dr. Eduardo Mazzucchi Division of Urology Medical School, University of Sao Paulo, USP

Sao Paulo, SP, Brazil E-mail:mazuchi@terra.com.br

\section{EDITORIAL COMMENT}

This paper compared 2 methods of drainage for perinephric abscess: percutaneous versus open surgical drainage. The authors found that open drainage was associated with higher cure rates, lower recurrence rates and shorter hospital stay, particularly in patients with multilocular abscess. They correctly emphasized that percutaneous drainage is the first line option of treatment in severely ill patients with perinephric unilocular abscess. Currently, perinephric abscesses are uncommon in most urology units (1) and 
since 1998, in my unit, we have seen fewer cases. It is therefore refreshing to read about the experience of a unit where this clinical entity remains a common clinical problem.

A major flaw of this paper is that it was a retrospective analysis and the patients had many variables as shown in tables 1 and 2. For example, the authors stated that percutaneous drainage was chosen for severely ill patients who could not withstand general anesthesia. The longer hospital stay and higher recurrence rates in patients subjected to percutaneous drainage could be attributed to the fact that they presented with worse initial disease, compared to those who underwent open drainage. The location of the abscesses, their configuration, etc., are variables that may affect the success of percutaneous drainage. However, the paper contains some useful guidelines (Figure-1) for units that do not have adequate experience in the management of these patients. It must be stated that a randomized controlled study will be difficult to carry out in patients with perinephric abscess because of its declining incidence, the variable ways in which patients can present and many other variables in individual patients with the disease. A last point that is worth emphasizing is that, as in patients with emphysematous pyelonephritis, a differential renogram test should be carried out as soon as possible in patients with moderate to severe perinephric abscess (1). Patients found to have poorly functioning kidneys $(<15 \%)$ or non-functioning kidneys are best served by nephrectomy as soon as possible rather than open or prolonged percutaneous drainage. Nephrectomy has been shown to reduce morbidity and mortality in patients with perinephric abscess (1).

\section{REFERENCE}

1. Abdul-Halim H, Kehinde EO, Abdeen S, Lashin I, Al-Hunayan AA, Al-Awadi KA: Severe emphysematous pyelonephritis in diabetic patients: diagnosis and aspects of surgical management. Urol Int. 2005; 75: 123-8.

Dr. Elijah O. Kehinde

Professor of Urological Surgery Division of Urology, Department of Surgery Faculty of Medicine, Kuwait University Safat, Kuwait E-mail:ekehinde@hsc.edu.kw

\section{REPLY BY THE AUTHORS}

We agree with all the comments of Dr. Eduardo Mazzucchi. Concerning the comments of Dr. Elijah O. Kehinde, we have to emphasis some points. First, the reference he mentioned is dealing with emphysematous pyelonephritis not perinephric abscess (PNA). Second, we recommended percutaneous drainage (PD) for PNA in patients with severe illness and also for patients with unilocular abscess cavity (as mentioned in figure 1 and in the discussion and conclusion). Lastly, we disagree with the comment that "non-functioning kidneys are best served by nephrectomy as soon as possible rather than open or prolonged percutaneous drainage." This can be true for nephrectomy in case of emphysematous pyelonephritis because it is a chronic inflammation, but PNA is an acute inflammatory condition. We still advise drainage of the abscess with either PCD or open surgery. Then nephrectomy for poorly functioning kidney can be delayed until improvement of the patient's general condition and recovery from the toxemia of an acute abscess. 